\title{
Tuberculosis patients with unknown HIV status in the Netherlands: analysing underreporting and lack of testing
}

\author{
To the Editor:
}

The epidemics of tuberculosis (TB) and HIV are closely linked, as HIV infection is the main risk factor for progression from latent TB infection to active TB disease [1]. Globally, 11\% of the 10.4 million new TB cases in 2015 were HIV-infected, and 22\% of the 1.8 million TB deaths were among HIV-infected patients [2]. Despite all efforts made by collaborative TB/HIV programmes, less than half of all TB patients worldwide are estimated to be tested for HIV infection [2,3]. Only 19 of the 31 countries, representing a third of all TB patients in the European Union (EU) and European Economic Area (EEA), report HIV status, with $67.6 \%$ of patients in these countries having a known HIV status [4]. Better information on the burden of TB/HIV co-infections will aid the planning and evaluation of TB/HIV prevention and control activities in various countries [5-8].

In the Netherlands, 800-900 people are diagnosed with TB annually (notification rate 5.1 per 100000 in 2015) [9]. The proportion of TB patients with known HIV status in the Netherlands Tuberculosis Register (NTR) has increased from $20 \%$ in 2006, but levelled off to around $50-60 \%$ within recent years, despite the National TB Control Plan and national guidelines recommendation of routine HIV testing, now in existence for a decade [10,11]. The proportion of patients known to be HIV-positive in 2011-2015 was $3.1 \%$ among all notified patients and 5.6\% among those with a known status.

We analysed whether an unknown HIV status in the NTR represented non-testing or non-reporting. In addition, we assessed factors associated with non-testing for HIV among TB patients in the Netherlands. We did this using an anonymised dataset of all TB patients notified in 2015 to the NTR (updated until May 24, 2016). Data on HIV variables included whether an HIV test was done, the result of the test, or the reason for not testing. We selected the TB patients without an HIV test result from the database and requested the TB departments of the Municipal Public Health Services (MPHS) to review their patient records on HIV test results. In cases of an in-hospital diagnosis, we requested the MPHS to contact the clinical specialist to retrieve HIV information from hospital records. The various MPHS were requested to update additional information on HIV status in the NTR. A new update of the NTR data was retrieved on October 17, 2016.

In 2015,865 patients were notified in the NTR. At baseline, the HIV status of 525 patients $(60.7 \%)$ had been recorded in the NTR, of whom 35 (6.7\%) were HIV-positive. After our intervention, 16 out of the total of 23 MPHS updated information, and an additional 108 patients (20.6\% more) had a known HIV status, giving a total of 633 patients $(73.2 \%)$ with a known HIV status. Known HIV testing varied widely between the 23 MPHS, i.e. between $11.1 \%$ and $90.4 \%$ at baseline, and between $22.2 \%$ and $98.3 \%$ after our intervention. No additional HIV-positive patients were identified. Of patients with an unknown HIV status, 109 patients (12.6\%) were not tested ( 80 not offered, eight patients refused and for 21 patients, the reason for not testing was unknown), and in 123 patients (14.2\%), it was not known whether an HIV test was done.

The characteristics of all TB patients, and those with known HIV status, those not tested for HIV and those for which it was unknown whether they were tested for HIV, are provided in table 1. After adjustment for other covariates, female patients (OR 1.6, 95\% CI 1.0-2.5), patients <15 years (OR 4.9, 95\% CI 1.9-12.3), patients $\geqslant 65$ years of age (OR 4.5, 95\% CI 2.3-8.9), those detected through contact

@ERSpublications

Few HIV-co-infected TB patients are missed despite suboptimal testing and reporting in the Netherlands http://ow.ly/VT9230fPJpi

Cite this article as: Arnoldussen M, Schimmel H, Op de Coul E, et al. Tuberculosis patients with unknown HIV status in the Netherlands: analysing underreporting and lack of testing. Eur Respir J 2017; 50: 1701257 [https://doi.org/10.1183/13993003.01257-2017]. 
TABLE 1 Characteristics of tuberculosis (TB) patients notified in the Netherlands in 2015 by HIV status after the study intervention

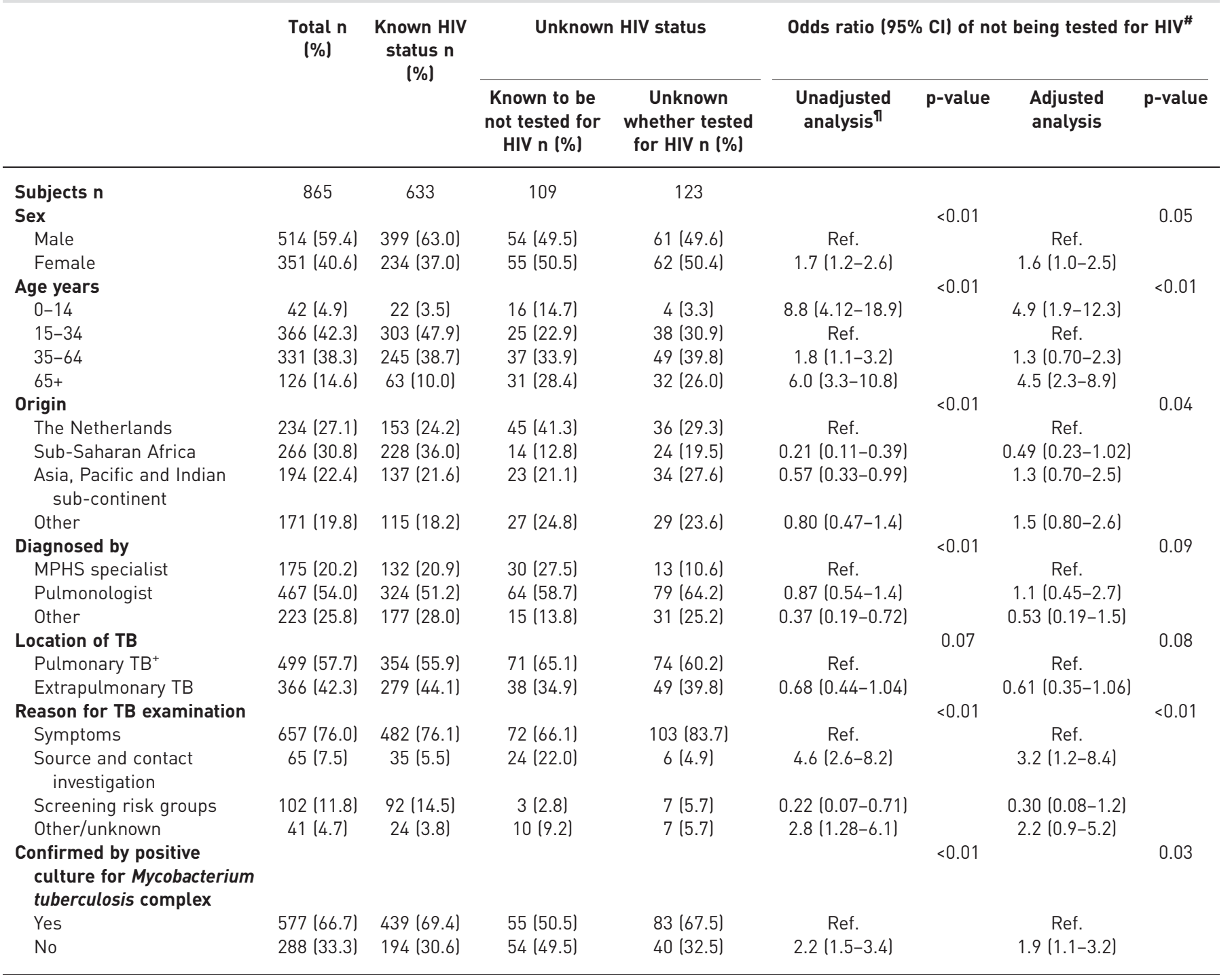

MPHS: Municipal Public Health Service. " : compared to having known HIV status; " : the variables duration of residence in the Netherlands, reason for immigration, legal status and previous TB diagnosis have been taken into account in initial univariate analysis, but were excluded from the analysis, owing to a lack of association; ${ }^{+}$: including 131 patients with both pulmonary and extrapulmonary TB.

investigation (OR 3.2, 95\% CI 1.2-8.4) and those without culture confirmation of the disease (OR 1.9, 95\% CI 1.1-3.2) were more frequently not tested for HIV.

Children ( $0-14$ years) and the elderly ( $\geqslant 65$ years) have a lower pre-test probability of HIV infection based on historical NTR data. During the years 2006-2015, $0.9 \%$ of the children and $0.6 \%$ of the elderly with TB had an HIV co-infection reported in the NTR, whereas this proportion was $4.4 \%$ for patients aged 15-64 years. Thus, we can assume that those tested would have a higher average pre-test probability than those not tested. This is confirmed by the finding that although our study yielded a $21 \%$ increase in the number of patients with a known HIV status, none of these patients had a positive HIV test.

The large differences in known HIV testing coverage per MPHS suggest that practices of HIV testing, and recording and reporting of the results are highly variable in the Netherlands, which is consistent with observations in another study in the UK [12]. We noted that HIV test results were sometimes known by the MPHS, but not entered in the NTR. This non-reporting can partly be explained by the manner in which the notification system is organised in the Netherlands, which promotes completion of the first part within 2 months, and includes the option "HIV status unknown". The MPHS sometimes receive HIV test 
results several months after notification, e.g. in a correspondence letter from the clinical specialist; thus, the "HIV status unknown" result is not always updated in the NTR. Clinical specialists should be encouraged to report HIV test results to the MPHS early, to ensure optimum treatment. The MPHS should also check the completeness of the data, including that of the HIV test results, at the end of treatment in the NTR. We also noted that HIV test results were sometimes known by the clinical specialists, but not communicated to the MPHS.

Previous research has shown that a TB/HIV co-infection is less likely to be missed by using an opt-out approach for HIV testing, compared to individual opt-in testing [13], since individual risk assessment will no longer influence the choice of whether or not to perform an HIV test [14]. In that way, HIV screening would be more of a routine procedure and could eliminate missed opportunities for the early diagnosis of HIV.

Our study findings have led to recommendations to improve the interoperability between systems, ensuring that all HIV test results are entered into the national TB register. It has also supported the development of arrangements between clinical specialists and the various MPHS, including communication of HIV test results, e.g. through standard HIV test reporting. Based on our results, we conclude that knowledge, attitudes and practices of clinical and public health specialists on HIV testing of TB patients should receive increased attention in professional education and re-training programmes. Our study also indicates that professionals frequently do not offer an HIV test to patients with a perceived nil pre-test probability. For example, undiagnosed HIV infection in children born in the Netherlands is extremely rare, because according to an opt-out testing policy, universal HIV screening has been implemented in pregnant women for over a decade, with only $0.2 \%$ refusals of the HIV test [15]. Therefore, it would be beneficial for guideline committees to take the local or country-specific situations into account and adopt corresponding tailored recommendations, or clearly explain the underlying scientific evidence for potential counter-intuitive recommendations.

In conclusion, our findings show that few HIV-co-infected TB patients are missed, despite suboptimal testing and reporting in the Netherlands. Nevertheless, this study has provided suggestions for both structural and practical improvements in TB/HIV surveillance that should become evident in the years to come.

Maike Arnoldussen ${ }^{1}$, Henrieke Schimmel ${ }^{2}$, Eline Op de Coul ${ }^{2}$, Susan van den $\mathrm{Hof}^{3,4}$ and Gerard de Vries ${ }^{2,3}$

${ }^{1}$ Faculty of Health, Medicine and Life Sciences, Maastricht University, Maastricht, The Netherlands. ${ }^{2}$ Centre for Infectious Disease Control, National Institute for Public Health and the Environment (RIVM), Bilthoven, The Netherlands. ${ }^{3}$ KNCV Tuberculosis Foundation, The Hague, The Netherlands. ${ }^{4}$ Dept of Global Health, Academic Medical Center; and Amsterdam Institute for Global Health and Development, Amsterdam, The Netherlands.

Correspondence: G. de Vries, KNCV Tuberculosis Foundation, PO Box 146, 2501 CC Den Haag, The Netherlands. E-mail: gerard.devries@kncvtbc.org

Received: Jan 152017 | Accepted after revision: Aug 242017

Conflict of interest: None declared.

Acknowledgement: The authors would like to thank the public health nurses and physicians of the TB departments of the Municipal Public Health Services who participated in this study.

\section{References}

1 Martinson NA, Hoffmann CJ, Chaisson RE. Epidemiology of tuberculosis and HIV. Proc Am Thorac Soc 2011; 8: 288-293.

2 World Health Organization. Global Tuberculosis Control: WHO report 2016 (WHO/HTM/TB/2016.13). Geneva, World Health Organization, 2016.

3 WHO policy on collaborative TB/HIV activities: guidelines for national programmes and other stakeholders. Geneva, World Health Organization, 2012.

4 European Centre for Disease Prevention and Control/WHO Regional Office for Europe. Tuberculosis surveillance and monitoring in Europe 2016. Stockholm, European Centre for Disease Prevention and Control, 2016.

5 Scholten JN, de Vlas SJ, Zaleskis R. Under-reporting of HIV infection among cohorts of TB patients in the WHO European Region, 2003-2004. Int J Tuberc Lung Dis 2008; 12: Suppl. 1, S85-S91.

6 Kruijshaar ME, Pimpin L, Abubakar I, et al. The burden of TB-HIV in the EU: how much do we know? A survey of surveillance practices and results. Eur Respir J 2011; 38: 1374-1381.

7 Pimpin L, Drumright LN, Kruijshaar ME, et al. Tuberculosis and HIV co-infection in European Union and European Economic Area countries. Eur Respir J 2011; 38: 1382-1392.

8 van der Werf MJ, Ködmön C, Zucs P, et al. Tuberculosis and HIV coinfection in Europe: looking at one reality from two angles. AIDS 2016; 30: 2845-2853.

9 Tuberculose in Nederland 2015 - Surveillance rapport inclusief rapportage monitoring van interventies. Bilthoven, RIVM, 2016. Available from: www.rivm.nl/Documenten_en_publicaties/Wetenschappelijk/Rapporten/2011/juli/ Nationaal_plan_tuberculosebestrijding_2011_2015_Inhoudelijke_kaders

10 National Tuberculosis Control Plan 2011-2015. Bilthoven, RIVM, 2010. 
11 CPT-richtlijn Tuberculose-HIV. Den Haag, Commissie voor Praktische Tuberculosebestrijding, KNCV Tuberculosefonds, 2016. Available from: www.kncvtbc.org/uploaded/2016/01/3.14_tuberculose.pdf

12 Rodger AJ, Story A, Fox Z, et al. HIV prevalence and testing practices among tuberculosis cases in London: a missed opportunity for HIV diagnosis? Thorax 2010; 65: 63-69.

13 Long R, Niruban S, Heffernan C, et al. A 10-year population based study of "opt-out" HIV testing of tuberculosis patients in Alberta, Canada: national implications. PLOS ONE 2014; 9: e98993.

14 TB Elimination. Recommendations for Human Immunodeficiency Virus (HIV) Screening in Tuberculosis (TB) Clinics. Atlanta, Centres for Disease Control and Prevention, 2012.

15 Op de Coul EL, Hahné S, van Weert YW, et al. Antenatal screening for HIV, hepatitis B and syphilis in the Netherlands is effective. BMC Infect Dis 2011; 11: 185.

Copyright @ERS 2017 